\title{
Shape design for polymer spin packs: modeling, optimization and validation
}

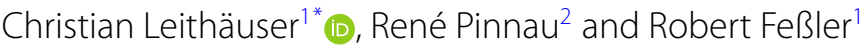

${ }^{\text {"Correspondence: }}$

christian.leithaeuser@itwm.fraunhofer.de

${ }^{1}$ Fraunhofer Institute for Industrial

Mathematics ITWM, Kaiserslautern,

Germany

Full list of author information is

available at the end of the article

\begin{abstract}
A shape optimization approach for the design of cavities with a specified wall shear stress profile is presented. Applications are the design of spin pack geometries with low and uniform residence times and without dead spaces to prevent polymer degradation for sensitive materials. The optimization uses a Surrogate Model based on the Newtonian Stokes equation as a simplification. An indirect objective based on wall shear stress is used to improve the residence time. The results are then validated on a realistic spin pack with the Full Model based on the non-Newtonian Navier-Stokes equation.
\end{abstract}

Keywords: Shape optimization; Computational fluid dynamics; Navier-Stokes equation; Non-Newtonian fluid; Approximate controllability

\section{Introduction}

Polymer spin packs are widely used for the production of synthetic fibers and non-woven materials. Polymer melt is extruded through a pipe into the spin pack geometry where it is first distributed along the whole cross-sectional area before it passes several layers of filter material and is finally spun into fibers by the spinneret plate which consists of a large number of very small nozzles. The whole spin pack is heated in order to prevent premature solidification of the melt. However, the influence of heat can lead to polymer degradation if the residence times are too long. For sensitive polymers with interesting properties this issue can be the limiting factor which prevents innovations due to the fact that spinning is not possible. This can be resolved by designing special spin packs with low residence time profiles. An important part in the spin pack design is the cavity which distributes polymer from the inlet pipe onto the whole cross-sectional area. This part of the geometry is in particular vulnerable for dead spaces and regions with slow flow velocities where degradation can take place. An indirect objective based on the wall shear stress is used to improve the residence time. The idea is that problematic regions usually occur in close proximity to the walls. In general a low wall shear stress coincides with a slow velocity zone close to the wall. On the other hand being able to design cavities with a sufficiently high level of stress throughout its wall is an effective tool against dead spaces and large residence times and thus against polymer degradation.

A spin pack is geometrically complex (see Fig. 1) and most polymers are non-Newtonian. Typically, commercial simulation software is used for the simulation, which makes derivative based shape optimization difficult to handle. Therefore, a surrogate model approach

(c) The Author(s) 2018. This article is distributed under the terms of the Creative Commons Attribution 4.0 International License (http://creativecommons.org/licenses/by/4.0/), which permits unrestricted use, distribution, and reproduction in any medium, provided you give appropriate credit to the original author(s) and the source, provide a link to the Creative Commons license, and indicate if changes were made. 


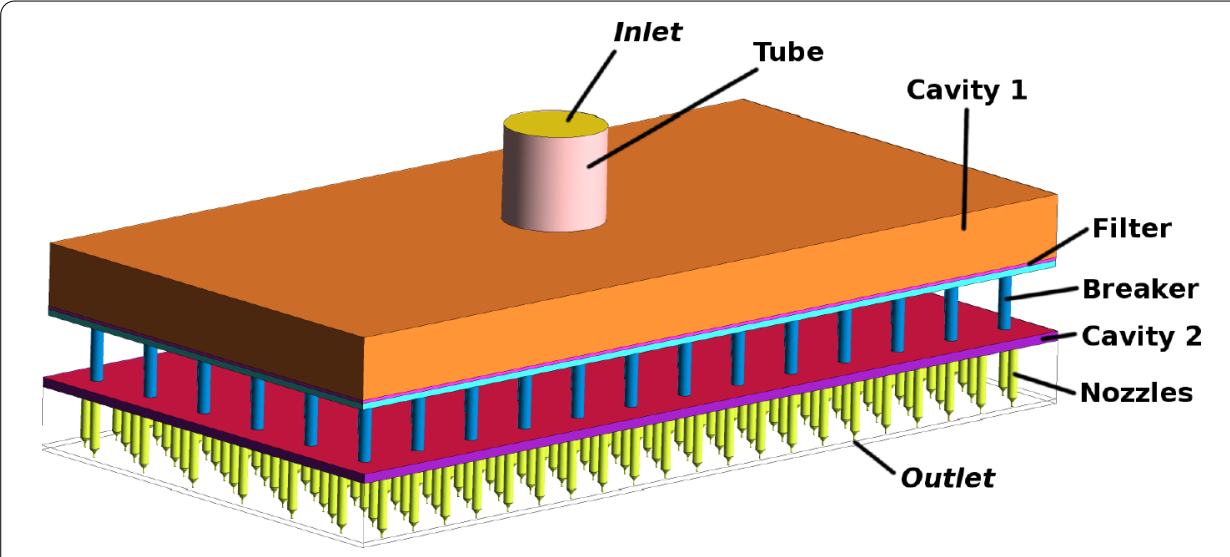

Figure 1 Geometry of the reference spin pack design

is used to optimize specific parts of the spin pack. The improved design is then validated with the complex model.

In Sect. 2 the two models are introduced: The Full Model is used for validation and is based on the non-Newtonian Navier-Stokes equation. It considers the whole geometry of the spin pack and pathlines are traced to evaluate the distribution of residence times. The Surrogate Model on the other hand is based on the Newtonian Stokes equation. It only considers the geometry of the spin pack cavity and wall shear stress is used as an indirect objective instead of residence time. The numerical approach for solving the shape optimization problem based on the Surrogate Model is derived in Sect. 2.3 and optimized cavities with uniform wall shear stress are presented in Sect. 3.1. These cavities are then validated with the Full Model in Sect. 3.2 in a realistic setting.

Further numerical and theoretical results on the shape optimization of polymer spin packs can be found in [1-4]. Another interesting application of a similar problem is studied in $[5,6]$. The authors use shape optimization to design aorto-coronaric bypasses and use wall shear stress as an optimization criterion.

\section{Methods}

A typical spin pack used for fiber production is shown in Fig. 1. Polymer enters through the Inlet, passes a short Tube and is distributed in Cavity 1 onto the Filter. Polymer passes the Breaker plate, which is basically a metal plate with a number of holes and its main purpose is to support the Filter. There is a second cavity (Cavity 2) before the material enters the Nozzles and is spun into fibers. The Nozzles consist of larger counterbores and then very small capillaries where the actual spinning takes place.

In the following we first introduce the Full Model based on the non-Newtonian NavierStokes equation which is used to compute the residence time of the polymer within the spin pack. After this the Surrogate Model based on the Newtonian Stokes equation is introduced. The Surrogate Model is used for the optimization of the geometry of Cavity 1 . See Table 1 for a comparison of the two models. The detailed simplifications and their justifications are given later in the Sect. 2.2. 
Table 1 Comparison between Full and Surrogate Model

\begin{tabular}{lll}
\hline & Full Model & Surrogate Model \\
\hline PDE & Navier-Stokes & Stokes \\
Viscosity & non-Newtonian & Newtonian \\
Geometry & whole spin pack & Cavity 1 (Filter in boundary condition) \\
Objective & residence time & wall shear stress \\
\hline
\end{tabular}

\subsection{Full model: non-Newtonian Navier-Stokes}

Let $\Omega \subset \mathbb{R}^{3}$ be the geometry of the spin pack. The flow is modeled using the stationary Navier-Stokes equation

$$
\begin{aligned}
& -\frac{\eta(\dot{\gamma}, T)}{\rho} \Delta \mathbf{u}+(\mathbf{u} \cdot \nabla) \mathbf{u}+\frac{1}{\rho} \nabla p=\mathbf{F} \quad \text { in } \Omega, \\
& \operatorname{div} \mathbf{u}=0 \quad \text { in } \Omega
\end{aligned}
$$

with velocity $\mathbf{u}$, pressure $p$ and density $\rho$. The viscosity $\eta(\dot{\gamma}, T)$ depends on the shear rate $\dot{\gamma}$ and temperature $T$. The temperature is assumed to be constant due to the controlled heating of the spin pack block. The source term $\mathbf{F}$ is set to zero everywhere except in the Filter where it is used to model a porous medium.

The shear rate is defined through the rate-of-deformation tensor $\bar{D}$ by (see [7])

$$
\dot{\gamma}=\sqrt{\frac{1}{2} \bar{D}: \bar{D}}
$$

and the tensor is given by

$$
\bar{D}=\left(\partial_{i} u_{j}+\partial_{j} u_{i}\right)_{i j}
$$

In order to model the viscosity a Cross model $[8$, Ch. 3.6] is used

$$
\eta(\dot{\gamma}, T)=H(T) \frac{\eta_{0}}{1+(\lambda \dot{\gamma})^{1-n}}
$$

with zero-shear-rate viscosity $\eta_{0}$, time constant $\lambda$ and power-law index $n$. The temperature dependence is modeled through an Arrhenius law by

$$
H(T)=\exp \left(\alpha\left(\frac{1}{T}-\frac{1}{T_{\alpha}}\right)\right)
$$

with activation energy $\alpha$ and reference temperature $T_{\alpha}$.

The Filter is modeled as a porous medium by adding a source term to the momentum equation [7]

$$
\begin{aligned}
& \mathbf{F}=C_{\text {filter }} \frac{1}{2} \rho|\mathbf{u}| \mathbf{u} \quad \text { in } \Omega_{\text {filter }}, \\
& \mathbf{F}=0 \quad \text { in } \Omega \backslash \Omega_{\text {filter }},
\end{aligned}
$$

where $\Omega_{\text {filter }}$ is the filter part of the computational domain (see Fig. 1). Typically the filter screens used in polymer spin packs are woven meshes made from metal wire and the pore 
Figure 2 Sketch of a geometry of the Surrogate Model with boundary partition



size as well as the number of pores per area are known. If one assumes equal flow rates per filter area it is possible to estimate the inertial loss coefficient $C_{\text {filter }}$ from simulating the flow through a single pore. This assumption of uniform flow rates often holds because the Filter generates a significant pressure drop compared to its surroundings (cf. Fig. 9).

Once the flow has been computed the residence time needs to be quantified. This is done by evaluating the residence time for a finite number of pathlines through the spin pack. Typically, we use the pathlines which end in the center of every capillary. The distribution of the residence times for the individual pathlines is used to compare the residence time distribution for different spin pack designs (cf. Fig. 8). The residence time for a single pathline is computed in the following way: Let $\mathbf{x}_{\text {out }}$ be a point on the outlet, for example in the center of a capillary. The pathline is computed in reverse order by solving the ODE

$$
\begin{aligned}
& \dot{\mathbf{x}}(t)=-\mathbf{u}(\mathbf{x}), \\
& \mathbf{x}(0)=\mathbf{x}_{\text {out }} .
\end{aligned}
$$

Note, that the sign in front of the velocity $\mathbf{u}$ is negative because the pathline is traced in reverse direction from outlet to inlet. The residence time for this individual pathline is then the time $t$ at which the pathline reaches the inlet boundary.

\subsection{Surrogate model: Newtonian Stokes}

The goal is now to optimize the geometry such that the residence time distribution is short and with small deviations. To do this a number of simplifications leading to the Surrogate Model are introduced in the following.

Simplification 1: geometry. Instead of the full spin pack geometry depicted in Fig. 1 we only consider Cavity 1 for our Surrogate Model. This is signified because typically most of the residence time is spent in this cavity. The boundary decomposed into an inlet part $\Gamma^{\text {in }}$, a wall part $\Gamma^{\text {wall }}$ and an outlet part $\Gamma^{\text {out }}$ (see Fig. 2).

Furthermore, the Filter sitting below the cavity is accounted for in the outlet boundary condition through a Darcy law [9, Eq. 1.2] which yields a relation between normal velocity and pressure:

$$
n_{\text {out }}(\mathbf{n} \cdot \mathbf{u})=-\frac{k_{\text {out }}}{\eta} \frac{p-p_{\text {amb }}}{L_{\text {out }}} \quad \text { on } \Gamma^{\text {out }}
$$

with the porosity $n_{\text {out }}$, the permeability $k_{\text {out }}$, the ambient pressure $p_{\text {amb }}$ and the thickness of the filter $L_{\text {out }}$. It is important to consider the filter to get the correct velocity at the outlet. The filter typically generates a much higher pressure drop than the friction of the cavity. This basically results in equal flow rates throughout the whole outflow boundary.

Simplification 2: Newtonian viscosity. Typical polymers used for fiber production are non-Newtonian and shear thinning plays a role, i.e., higher shear rates lead to a lower 
viscosity. However, for most applications the shear thinning only occurs in the fine capillaries. The shear rates in the distributor cavities usually lie in the zero shear rate limit of the Cross model [8, Ch. 3.6] used to represent the viscosity (cf. Fig. 6). Thus we use a constant viscosity

$$
\eta(\dot{\gamma}, T)=\eta=\text { const }
$$

which is independent of the shear rate.

Simplification 3: Stokes equation. Inertia does not play a role for the flow within the spin pack cavity, due to the high viscosity compared to the low velocities. Therefore, the Navier-Stokes equation can be replaced by the Stokes equation. The previous simplification lead to the following problem:

$$
\begin{aligned}
& -\eta \Delta \mathbf{u}+\nabla p=0 \quad \text { in } \Omega, \\
& \operatorname{div} \mathbf{u}=0 \quad \text { in } \Omega, \\
& \mathbf{u}=\mathbf{n} u_{0} \quad \text { on } \Gamma^{\text {in }}, \\
& \mathbf{u}=0 \quad \text { on } \Gamma^{\text {wall }}, \\
& c_{\text {out }}(\mathbf{n} \cdot \mathbf{u})+p=0 \quad \text { on } \Gamma^{\text {out }}, \\
& \mathbf{n} \times \mathbf{u}=0 \quad \text { on } \Gamma^{\text {out }},
\end{aligned}
$$

where $u_{0}$ is a given inflow profile and $\mathbf{n}$ the outward pointing normal vector. With

$$
c_{\text {out }}=\frac{n_{\text {out }} \eta L_{\text {out }}}{k_{\text {out }}}
$$

and w.l.o.g. $p_{\mathrm{amb}}=0$ the outflow boundary condition agrees with (6). The well-posedness of the Stokes problem for these specific boundary conditions follows from [10, Prop. 4.7].

Simplification 4: wall shear stress objective. We use a cost function based on the wall shear stress as an indirect criterion to optimize the residence time. The reason is that the wall shear stress

$$
\sigma=\eta|\nabla \times \mathbf{u}| \quad \text { on } \Gamma^{\text {wall }}
$$

can be directly evaluated from the flow in contrast to the residence time which involves an additional equation (5). Furthermore, when dealing with (5) it can happen that a pathline does not reach the end due to numerical reasons. This leads to discontinuities in the cost function and poses a problem for a gradient-based optimization approach.

The idea for using the wall shear stress is the following: Dead spaces and regions with slow flow velocities where degradation can take place usually occur in close proximity to the walls. A low wall shear stress coincides with a slow velocity zone. On the other hand a sufficiently high level of stress throughout the wall prevents dead spaces and reduces the residence time. 
This motivation leads to the following shape optimization problem:

$$
\min _{\substack{\Omega \\ \text { subject to (7) }}} J(\Omega)=\int_{\Gamma^{\text {wall }}}\left(\eta|\nabla \times \mathbf{u}|-\sigma_{d}\right)^{2} d s,
$$

where $\sigma_{d}$ is a sufficiently high target wall shear stress.

\subsection{Numerical shape optimization for the surrogate model}

Let us derive the shape optimization approach for the Surrogate Model (8).

Geometry variations. Starting from any admissible domain $\Omega_{0} \subset \mathbb{R}^{3}$ of class $C^{1,1}$ we want to compute a gradient $\mathbf{V}_{g}$ which enables us to use a gradient descent approach for solving the shape optimization problem. Knowing the gradient we can perform a descent step towards the domain $\Omega_{-s \mathbf{V}_{g}}=\left(\mathrm{Id}-s \mathbf{V}_{g}\right)\left(\Omega_{0}\right)$ for some step size $s>0$. Let

$$
\begin{aligned}
& \mathcal{V}^{1}:=\left\{\mathbf{V} \in C^{1,1}\left(\mathbb{R}^{3}, \mathbb{R}^{3}\right) ;\left.\mathbf{V}\right|_{\Gamma_{0}^{\text {in }} \cup \Gamma_{0}^{\text {out }}}=0\right\}, \\
& \Theta^{1}:=\left\{\theta \in \mathcal{V}^{1} ;\|\theta\|_{C^{1,1}\left(\mathbb{R}^{3}, \mathbb{R}^{3}\right)}<\frac{1}{2}\right\} .
\end{aligned}
$$

We define $\Omega_{\theta}:=(\operatorname{Id}+\theta)\left(\Omega_{0}\right)$ which is again of class $C^{1,1}$ (see Remark 1$)$ and consider the problem

$$
\begin{aligned}
& -\mu \Delta \mathbf{u}(\theta)+\nabla p(\theta)=0 \quad \text { in } \Omega_{\theta}, \\
& \operatorname{div} \mathbf{u}(\theta)=0 \quad \text { in } \Omega_{\theta}, \\
& \mathbf{u}(\theta)=\mathbf{u}_{0} \quad \text { on } \Gamma_{\theta}^{\text {in }}, \\
& \mathbf{u}(\theta)=0 \quad \text { on } \Gamma_{\theta}^{w}, \\
& c_{\text {out }}(\mathbf{n} \cdot \mathbf{u}(\theta))+p(\theta)=0 \quad \text { on } \Gamma_{\theta}^{\text {out }}, \\
& \mathbf{u}(\theta) \times \mathbf{n}=0 \quad \text { on } \Gamma_{\theta}^{\text {out }}
\end{aligned}
$$

with wall shear stress

$$
\sigma(\theta)=\left.\mu|\nabla \times \mathbf{u}(\theta)|\right|_{\Gamma_{\theta}^{w}}=\left.\mu \sqrt{(\nabla \times \mathbf{u}(\theta)) \cdot(\nabla \times \mathbf{u}(\theta))}\right|_{\Gamma_{\theta}^{w}} .
$$

Remark 1 For $\theta \in \Theta^{1}$ it is implied by [11] that $\operatorname{Id}+\theta: \mathbb{R}^{3} \rightarrow \mathbb{R}^{3}$ is an invertible $(1,1)$ diffeomorphism and thus $\Omega_{\theta}=(\operatorname{Id}+\theta)\left(\Omega_{0}\right)$ is also of class $C^{1,1}$. Then, a regularity argument similar to [12] would yield $\mathbf{u}(\theta) \in\left[H^{2}\left(\Omega_{\theta}\right)\right]^{3}$, thus $\sigma(\theta) \in L^{2}\left(\Gamma_{\theta}^{w}\right)$ by the Trace Theorem [13, Thm. 8.7] and the objective (8) is well-defined. However, the focus of the current paper lies in the application and we will not derive the regularity result for our specific set of boundary conditions (10). We will rather use Assumption 1 and derive the gradient in a purely formal way.

Remark 2 The $C^{1,1}$ regularity of the domain can probably be relaxed further: If should suffice if the wall boundary $\Gamma_{\theta}^{w}$ is $C^{1,1}$. Therefore, sharp corners between inlet/outlet and the wall should be allowed which is reasonable for the application (cf. Figs. 3 and 4). This is further signified by the numerical results. 


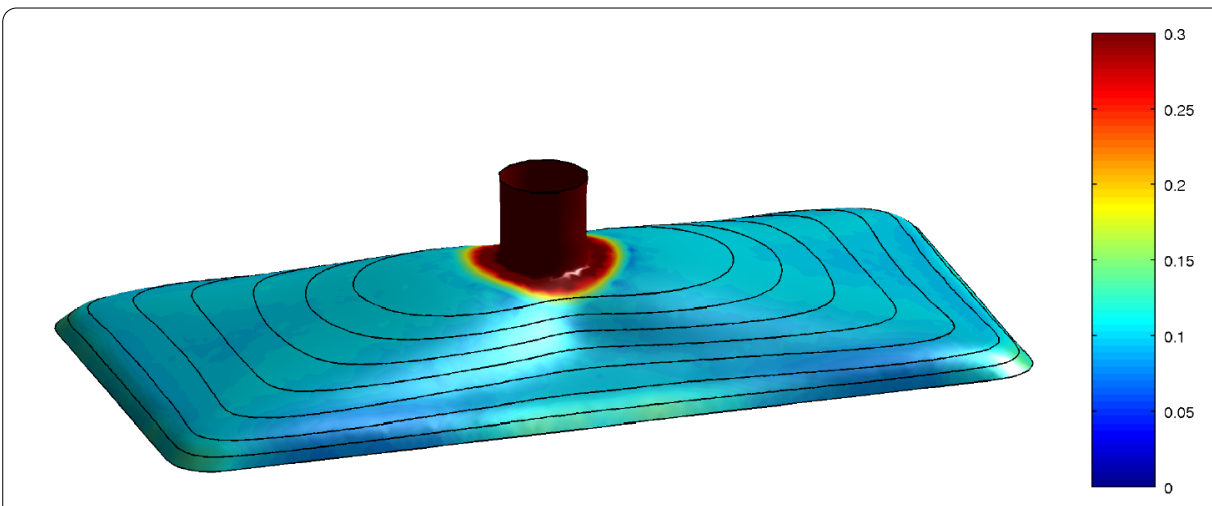

Figure 3 Cavity A: Optimal distributor geometry for a target wall shear stress of $\sigma_{d}=0.1$



Figure 4 Cavity B: Optimal distributor geometry for a target wall shear stress of $\sigma_{d}=0.2$

Sensitivity analysis. In order to compute the sensitivity we follow the optimize then discretize approach. Therefore, we need to differentiate the cost functional and the partial differential equation with respect to the shape, which requires the existence of the corresponding shape derivatives. However, the focus of this part is to derive a numerical method suited to solve the industrial problem, therefore, we omit the existence and regularity proofs for the shape derivatives and make the following assumption:

Assumption 1 Assume the existence of $\mathbf{u}(\theta) \in H^{2}\left(\Omega_{\theta}, \mathbb{R}^{3}\right), p(\theta) \in H^{1}\left(\Omega_{\theta}\right)$ and $\sigma(\theta) \in$ $L^{2}\left(\Gamma_{\theta}^{w}\right)$ for $\theta \in \Theta^{1}$ and the existence of the shape derivatives $\mathbf{u}^{\prime}\left(\Omega_{0} ; \mathbf{V}\right) \in H^{2}\left(\Omega_{0}, \mathbb{R}^{3}\right)$, $p^{\prime}\left(\Omega_{0} ; \mathbf{V}\right) \in H^{1}\left(\Omega_{0}\right)$ and $\sigma^{\prime}\left(\Gamma_{0} ; \mathbf{V}\right) \in L^{2}\left(\Gamma_{0}^{w}\right)$ for $\mathbf{V} \in \mathcal{V}^{1}$.

A crucial point in the proof would be to show the regularity of the solution of the Stokes problem which is not trivial due to the mixed boundary condition. If this regularity is provided by some means we can use the implicit function theorem to show the existence of the material derivatives which provide the existence of the shape derivatives. This general approach to proof the existence of shape derivatives for partial differential equations is shown in [11]. In [3] we have applied this approach to a problem similar to the Surrogate Model considered here. 
Definition 1 (Shape derivative [14]) For $m \geq 1$ let $y(s \mathbf{V}) \in H^{m}\left((\operatorname{Id}+s \mathbf{V}) \circ \Omega_{0}\right)$ for $s \in \mathbb{R}$ sufficiently small. Then, the shape derivative of $y$ in direction $\mathbf{V}$ is defined by

$$
y^{\prime}\left(\Omega_{0}, \mathbf{V}\right):=\lim _{s \rightarrow 0} \frac{1}{s}(y(s \mathbf{V}) \circ(\mathrm{Id}+s \mathbf{V})-y(0))-\nabla y(0) \cdot \mathbf{V} \in H^{m-1}\left(\Omega_{0}\right) .
$$

Furthermore, the shape derivative of $y$ restricted to the boundary is

$$
y^{\prime}\left(\Gamma_{0}, \mathbf{V}\right):=\left.y^{\prime}\left(\Omega_{0}, \mathbf{V}\right)\right|_{\Gamma_{0}}+\partial_{\mathbf{n}} y(0)(\mathbf{n} \cdot \mathbf{V}) \in H^{m-\frac{3}{2}}\left(\Gamma_{0}\right)
$$

For details and a more general definition of the shape derivatives we refer to [14].

The cost function

$$
J\left(\Omega_{\theta}\right)=\int_{\Gamma_{\theta}^{w}}\left(\sigma(\theta)-\sigma_{d}\right)^{2} d s
$$

is differentiated with respect to $\theta$. The derivative of a boundary integral is given in [14]. Applying this to (12) yields for the derivative in direction $\mathbf{V} \in \mathcal{V}^{1}$

$$
\begin{aligned}
d J(\mathbf{V}) & =\frac{d J\left(\Omega_{\theta}\right)}{d \theta}(\mathbf{V}) \\
& =\int_{\Gamma_{0}^{w}}\left(\left.\left(\sigma(\theta)-\sigma_{d}\right)^{2}\right|_{\Gamma_{\theta}^{w}}\right)^{\prime}\left(\Gamma_{0} ; \mathbf{V}\right) d s+\int_{\Gamma_{0}^{w}} \kappa(\mathbf{n} \cdot \mathbf{V})\left(\sigma(0)-\sigma_{d}\right)^{2} d s,
\end{aligned}
$$

where $\kappa$ denotes the curvature (see [14]).

We assume that the wall shear stress $\sigma(0)$ is nonzero on the wall boundaries. For geometries with one inflow and one outflow this is fulfilled automatically. For more complicated geometries we may have to exclude neighborhoods of stagnation points from the wall boundaries. If this assumption holds, we can derive the shape derivative of $\sigma(\theta)$ by

$$
\begin{aligned}
\sigma^{\prime}\left(\Gamma_{0} ; \mathbf{V}\right) & =\frac{\mu}{2 \sqrt{\nabla \times \mathbf{u}(0) \cdot \nabla \times \mathbf{u}(0)}}\left(\left.(\nabla \times \mathbf{u}(\theta) \cdot \nabla \times \mathbf{u}(\theta))\right|_{\Gamma_{\theta}^{w}}\right)^{\prime}\left(\Gamma_{0} ; \mathbf{V}\right) \\
& =\frac{\mu^{2}}{\sigma(0)} \nabla \times \mathbf{u}(0) \cdot\left(\nabla \times\left.\mathbf{u}(\theta)\right|_{\Gamma_{\theta}^{w}}\right)^{\prime}\left(\Gamma_{0} ; \mathbf{V}\right) \\
& =\frac{\mu^{2}}{\sigma(0)} \nabla \times \mathbf{u}(0) \cdot\left(\left.\left(\nabla \times \mathbf{u}^{\prime}\left(\Omega_{0} ; \mathbf{V}\right)\right)\right|_{\Gamma_{0}^{w}}+(\mathbf{n} \cdot \mathbf{V}) \partial_{\mathbf{n}}(\nabla \times \mathbf{u}(0))\right) .
\end{aligned}
$$

Thus (13) becomes

$$
\begin{aligned}
d J(\mathbf{V})= & \int_{\Gamma_{0}^{w}} 2 \mu^{2} \frac{\sigma(0)-\sigma_{d}}{\sigma(0)} \nabla \times \mathbf{u}(0) \cdot \nabla \times \mathbf{u}^{\prime}\left(\Omega_{0} ; \mathbf{V}\right) d s \\
& +\int_{\Gamma_{0}^{w}} 2 \mu^{2} \frac{\sigma(0)-\sigma_{d}}{\sigma(0)} \nabla \times \mathbf{u}(0) \cdot \partial_{\mathbf{n}}(\nabla \times \mathbf{u}(0))(\mathbf{n} \cdot \mathbf{V}) d s \\
& +\int_{\Gamma_{0}^{w}} \kappa\left(\sigma(0)-\sigma_{d}\right)^{2}(\mathbf{n} \cdot \mathbf{V}) d s .
\end{aligned}
$$


We need to deal with the first term of (15) which contains the shape derivative $\mathbf{u}^{\prime}\left(\Omega_{0} ; \mathbf{V}\right)$. As shown in [15] the shape derivative $\left(\mathbf{u}^{\prime}\left(\Omega_{0} ; \mathbf{V}\right), p^{\prime}\left(\Omega_{0} ; \mathbf{V}\right)\right)$ can be computed as the solution of

$$
\begin{aligned}
& -\mu \Delta \mathbf{u}^{\prime}\left(\Omega_{0} ; \mathbf{V}\right)+\nabla p^{\prime}\left(\Omega_{0} ; \mathbf{V}\right)=0 \quad \text { in } \Omega_{0}, \\
& \operatorname{div} \mathbf{u}^{\prime}\left(\Omega_{0} ; \mathbf{V}\right)=0 \quad \text { in } \Omega_{0}, \\
& \mathbf{u}^{\prime}\left(\Omega_{0} ; \mathbf{V}\right)=0 \quad \text { on } \Gamma_{0}^{\text {in }}, \\
& \mathbf{u}^{\prime}\left(\Omega_{0} ; \mathbf{V}\right)=-(\mathbf{n} \cdot \mathbf{V}) \partial_{\mathbf{n}} \mathbf{u}(0) \quad \text { on } \Gamma_{0}^{w}, \\
& c_{\text {out }}\left(\mathbf{n} \cdot \mathbf{u}^{\prime}\left(\Omega_{0} ; \mathbf{V}\right)\right)+p^{\prime}\left(\Omega_{0} ; \mathbf{V}\right)=0 \quad \text { on } \Gamma_{0}^{\text {out }}, \\
& \mathbf{u}^{\prime}\left(\Omega_{0} ; \mathbf{V}\right) \times \mathbf{n}=0 \quad \text { on } \Gamma_{0}^{\text {out }} .
\end{aligned}
$$

We introduce the adjoint variables $(\mathbf{v}, q)$ as solutions of the adjoint Stokes problem

$$
\begin{aligned}
& -\mu \Delta \mathbf{v}+\nabla q=0 \quad \text { in } \Omega_{0}, \\
& \operatorname{div} \mathbf{v}=0 \quad \text { in } \Omega_{0}, \\
& \mathbf{v}=0 \quad \text { on } \Gamma_{0}^{\text {in }}, \\
& \mathbf{n} \cdot \mathbf{v}=0 \quad \text { on } \Gamma_{0}^{w}, \\
& \mathbf{v} \times \mathbf{n}=2 \mu \frac{\sigma-\sigma_{d}}{\sigma}(\nabla \times \mathbf{u}(0)) \text { on } \Gamma_{0}^{w}, \\
& c_{\text {out }}(\mathbf{n} \cdot \mathbf{v})+q=0 \quad \text { on } \Gamma_{0}^{\text {out }}, \\
& \mathbf{v} \times \mathbf{n}=0 \quad \text { on } \Gamma_{0}^{\text {out }} .
\end{aligned}
$$

Then, we can derive through integration by parts

$$
\begin{aligned}
0= & \int_{\Omega_{0}}\left(-\mu \Delta \mathbf{u}^{\prime}\left(\Omega_{0} ; \mathbf{V}\right)+\nabla p^{\prime}\left(\Omega_{0} ; \mathbf{V}\right)\right) \cdot \mathbf{v} d x+\int_{\Omega_{0}} \operatorname{div} \mathbf{u}^{\prime}\left(\Omega_{0} ; \mathbf{V}\right) q d x \\
= & \int_{\Omega_{0}} \mu \nabla \times \mathbf{u}^{\prime}\left(\Omega_{0} ; \mathbf{V}\right) \cdot \nabla \times \mathbf{v} d x+\int_{\Gamma_{0}} \mu \nabla \times \mathbf{u}^{\prime}\left(\Omega_{0} ; \mathbf{V}\right) \cdot(\mathbf{v} \times \mathbf{n}) d s \\
& -\int_{\Omega_{0}} p^{\prime}\left(\Omega_{0} ; \mathbf{V}\right) \operatorname{div} \mathbf{v} d x+\int_{\Gamma_{0}} p^{\prime}\left(\Omega_{0} ; \mathbf{V}\right) \mathbf{n} \cdot \mathbf{v} d s \\
& +\int_{\Omega_{0}} \mathbf{u}^{\prime}\left(\Omega_{0} ; \mathbf{V}\right) \cdot \nabla q d x-\int_{\Gamma_{0}} \mathbf{n} \cdot \mathbf{u}^{\prime}\left(\Omega_{0} ; \mathbf{V}\right) q d s \\
= & -\int_{\Omega_{0}} \mu \mathbf{u}^{\prime}\left(\Omega_{0} ; \mathbf{V}\right) \cdot \Delta \mathbf{v} d x+\int_{\Gamma_{0}} \mu \nabla \times \mathbf{u}^{\prime}\left(\Omega_{0} ; \mathbf{V}\right) \cdot(\mathbf{v} \times \mathbf{n}) d s \\
& -\int_{\Gamma_{0}} \mu\left(\mathbf{u}^{\prime}\left(\Omega_{0} ; \mathbf{V}\right) \times \mathbf{n}\right) \cdot \nabla \times \mathbf{v} d s+\int_{\Gamma_{0}} \eta_{\text {out }} \mathbf{n} \cdot \mathbf{u}^{\prime}\left(\Omega_{0} ; \mathbf{V}\right) \mathbf{n} \cdot \mathbf{v} d s \\
& +\int_{\Omega_{0}} \mathbf{u}^{\prime}\left(\Omega_{0} ; \mathbf{V}\right) \cdot \nabla q d x-\int_{\Gamma_{0}} \eta_{\text {out }} \mathbf{n} \cdot \mathbf{u}^{\prime}\left(\Omega_{0} ; \mathbf{V}\right) \mathbf{n} \cdot \mathbf{v} d s \\
= & \int_{\Gamma_{0}} \mu \nabla \times \mathbf{u}^{\prime}\left(\Omega_{0} ; \mathbf{V}\right) \cdot(\mathbf{v} \times \mathbf{n}) d s-\int_{\Gamma_{0}} \mu\left(\mathbf{u}^{\prime}\left(\Omega_{0} ; \mathbf{V}\right) \times \mathbf{n}\right) \cdot \nabla \times \mathbf{v} d s,
\end{aligned}
$$


which yields the identity

$$
\begin{gathered}
\int_{\Gamma_{0}^{w}} 2 \mu^{2} \frac{\sigma(0)-\sigma_{d}}{\sigma(0)} \nabla \times \mathbf{u}(0) \cdot \nabla \times \mathbf{u}^{\prime}\left(\Omega_{0} ; \mathbf{V}\right) d s \\
=-\int_{\Gamma_{0}^{w}} \mu\left(\partial_{\mathbf{n}} \mathbf{u}(0) \times \mathbf{n}\right) \cdot \nabla \times \mathbf{v}(\mathbf{n} \cdot \mathbf{V}) d s
\end{gathered}
$$

Plugging this identity into (15) yields

$$
\begin{aligned}
d J(\mathbf{V})= & -\int_{\Gamma_{0}^{W}} \mu\left(\partial_{\mathbf{n}} \mathbf{u}(0) \times \mathbf{n}\right) \cdot \nabla \times \mathbf{v}(\mathbf{n} \cdot \mathbf{V}) d s \\
& +\int_{\Gamma_{0}^{w}} 2 \mu^{2} \frac{\sigma(0)-\sigma_{d}}{\sigma(0)} \nabla \times \mathbf{u}(0) \cdot \partial_{\mathbf{n}}(\nabla \times \mathbf{u}(0))(\mathbf{n} \cdot \mathbf{V}) d s \\
& +\int_{\Gamma_{0}^{w}} \kappa\left(\sigma(0)-\sigma_{d}\right)^{2}(\mathbf{n} \cdot \mathbf{V}) d s .
\end{aligned}
$$

Discretization. Following the optimize then discretize approach we us Taylor-Hood finite elements to discretize the partial differential equations. The implementation is done in COMSOL Multiphysics.

Using the space $\mathcal{V}^{1}$ for the numerics is not reasonable because it would require the projection of the gradient into a high order Sobolev space which has an embedding into $C^{1,1}$. For our approach numerical results have shown that $H^{2}$-regularity of the gradient is sufficiently smooth, even though there is no embedding from $H^{2}$ into $C^{1,1}$. Therefore, in the discrete problem the space $\mathcal{V}^{1}$ is replaced with

$$
\mathcal{H}_{k}^{2}:=\left\{\mathbf{V} \in H^{2}\left(\Omega_{k}, \mathbb{R}^{3}\right) ;\left.\mathbf{V}\right|_{\Gamma_{k}^{\text {in }} \cup \Gamma_{k}^{\text {out }}}=0\right\} .
$$

The discrete gradient is then obtained by projecting $d J(v)$ into $\mathcal{H}_{k}^{2}$ by solving

$$
\left(\mathbf{V}_{k}, v\right)_{H^{2}\left(\Omega_{k}\right)}=d J(v) \quad \text { for all } v \in \mathcal{H}_{k}^{2}
$$

with $\mathbf{V}_{k} \in \mathcal{H}_{k}^{2}$ where $(\cdot, \cdot)_{H^{2}\left(\Omega_{0}\right)}$ denotes the scalar product in $H^{2}$.

Gradient descent method. In the last section the gradient of the cost function was derived, which enables us to apply the gradient descent method to solve the shape optimization problem. A small change of notation is performed: In the following let $\Omega_{k}$ denote the domain of iteration $k$ of the gradient descent algorithm and the gradient at $\Omega_{k}$ is denoted by $\mathbf{V}_{k}$. Using this notation the gradient descent method is given in Algorithm 1. An Armijo rule (cf. [16]) was used to determine the step length, where $\beta, \gamma>0$ are proper constants. Note, that the $L^{2}$-norm was used for step size control and stopping criterion. However, with the state space $\mathbf{V}_{k} \in \mathcal{H}_{k}^{2}$ the $H^{2}$-norm might be a better choice. We do not expect that this would significantly change the results, but it might improve the convergence.

Moving the mesh. This section explains how the mesh is moved and what smoothing operator $S_{\mathcal{T}}$ is used. An important question is how the shape deformation $\Omega_{k+1}=$ (Id $\left.\beta^{j} \mathbf{V}_{k}\right)\left(\Omega_{k}\right)$ is carried out. In the current setup we work with a triangulation $\mathcal{T}_{k}$ of $\Omega_{k}$ by tetrahedral elements. Then we move every vertex $\xi \in \mathcal{T}_{k}$ to $\left(\operatorname{Id}-\beta^{j} \mathbf{V}_{k}\right)(\xi)$ to generate the new mesh $\mathcal{T}_{k+1}$. In the case that any elements of the mesh are inverted we regenerate $\mathcal{T}_{k+1}$ while keeping the boundary triangulation. 


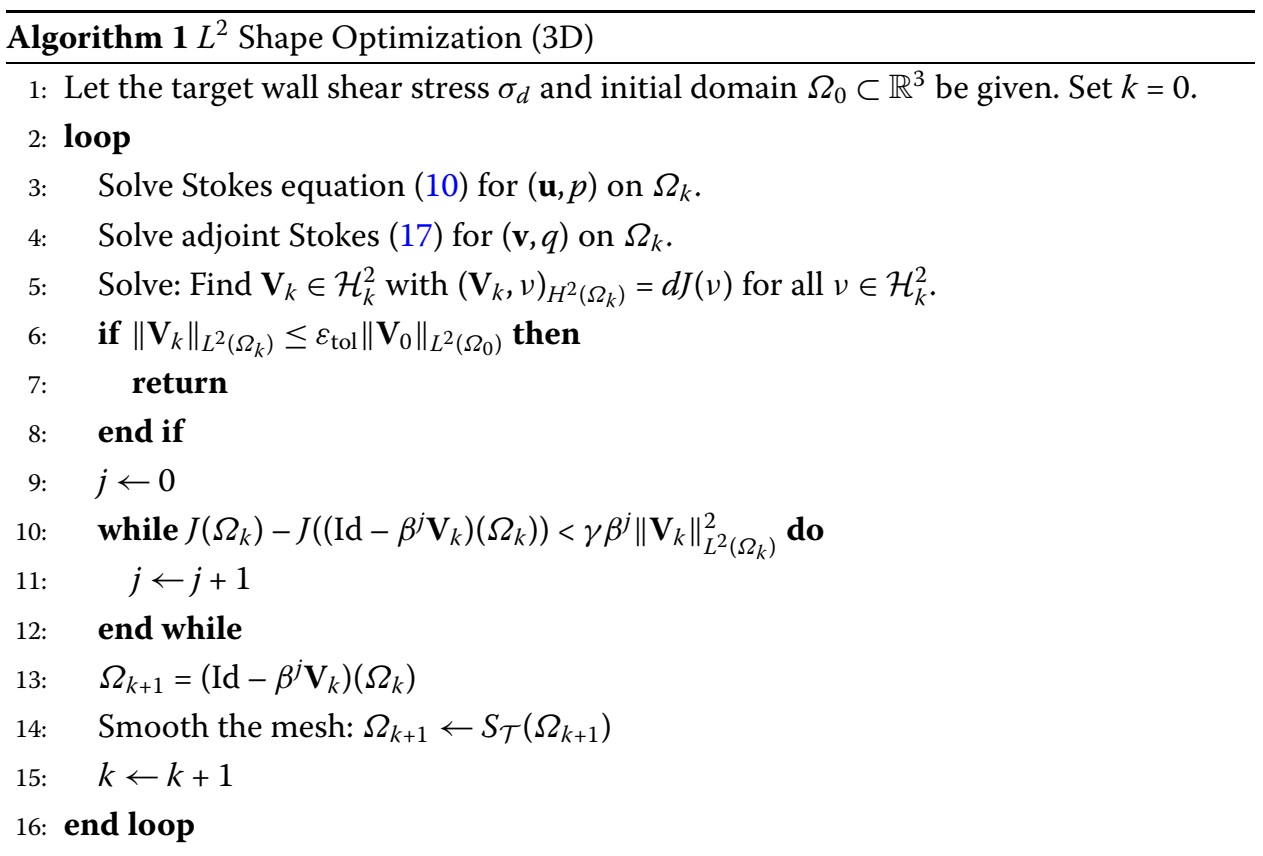

However, a problem is that the mesh tends to loose smoothness and becomes irregular. This has the effect that already after very few iterations the gradient fails to provide a proper descent direction and the algorithm stops. This is a common problem in shape optimization, as discussed in [15]. To overcome this we apply a smoothing operator $S_{\mathcal{T}}$ to the deformed mesh. The operator is applied to the boundary mesh and is able to recover a boundary shape with more regularity. Of course the in- and outflow boundaries are not effected by the smoothing. We use the implementation [17] which relies on [18]. Using the smoothing step leads to a good quality of the gradient and a stable gradient descent algorithm. See [2, Theorem 6.5] where we have shown the existence of an optimal control for a similar problem.

\section{Results and discussion}

In the following Algorithm 1 bases on the Surrogate Model is used to compute optimized cavities. These cavities are then tested with the Full Model. A dimensionless setting was used for the optimization, while the validation was done in a realistic setting with units given later.

\subsection{Shape optimization based on the surrogate model}

With the presented shape optimization approach it becomes possible to design cavities with a specific wall shear stress profile. Figures 3 and 4 show two cavities with a constant target wall shear stress of $\sigma_{d}=0.1$ and $\sigma_{d}=0.2$, respectively. Full details on the setup for deriving these shapes can be found in [2]. The wall shear stress is indicated by color and we see that it is possible to get a very close agreement between target and actual wall shear stress throughout wide parts of the geometries. This agrees with our theoretical results from [3] which suggests that the space of attainable wall shear stress profiles is rather large. Results on the convergence of Algorithm 1 are illustrated in Fig. 5. The convergence rates can likely be improved by using more advanced techniques then gradient descent 


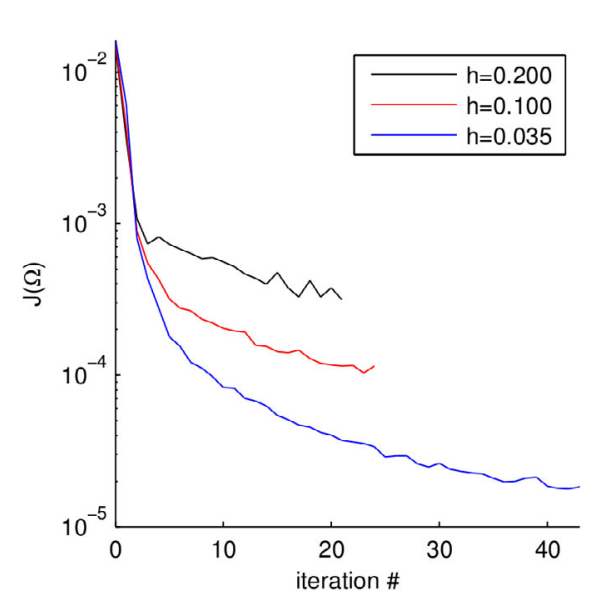

(a) Cost function $J\left(\Omega_{k}\right)$

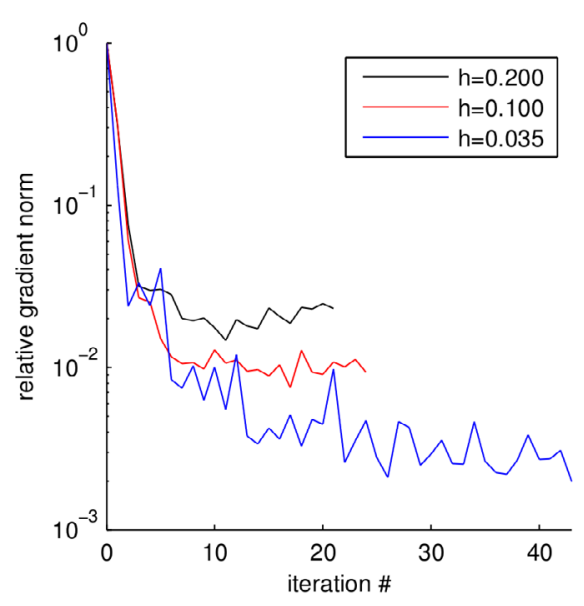

(b) Relative gradient norm

Figure 5 Convergence of the cost functional and the relative gradient norm using Algorithm 1 to compute Cavity A with different mesh sizes $h$

(cf. [19]). However, for our current applications this simple gradient descent approach has proven to be sufficient.

\subsection{Validation based on the full model}

While we have seen that the proposed shape optimization algorithm is able to design distributor cavities with a very uniform wall shear stress it remains to show how these cavities perform in a realistic application. In this section a typical reference spin pack design is compared to optimized designs. The reference design is shown in Fig. 1. In the reference design Cavity 1 is just a flat rectangular-shaped space which is quite common for many spin packs still in use today. However, this space is very vulnerable to dead spots which can then encourage polymer degradation due to long residence times. Therefore in the following Cavity 1 will be replaced by the two optimized cavities computed in Sect. 3.1 and results regarding residence time, wall shear stress and pressure drop are compared.

The commercial CFD software ANSYS ${ }^{\bullet}$ Fluent is applied to model the spin pack in this validation step.

Geometric setup, data and boundary conditions. The geometry of our reference spin pack with 279 nozzles is depicted in Fig. 1. The spacial dimensions of the bounding box are: $286 \mathrm{~mm} \times 146 \mathrm{~mm} \times 96 \mathrm{~mm}$ (length $\mathrm{x}$ width $\mathrm{x}$ height). In the following three geometries are compared:

- Reference: The reference design from Fig. 1.

- Optimized A: Cavity 1 of the reference design replaced with the optimized cavity from Fig. 3 (Cavity A).

- Optimized B: Cavity 1 of the reference design replaced with the optimized cavity from Fig. 4 (Cavity B).

These spin pack geometries can be seen from Fig. 7.

Polypropylene (PP) is used as the polymer. Raw data on the viscosity is taken from [20, Fig. 3.3-13] and the cross model was fitted to agree as good as possible. See Fig. 6 for a comparison of the literature data (markers) and the fitted cross model (lines). The fitted coefficients of the cross model are given in Table 2. Furthermore, a constant density of 


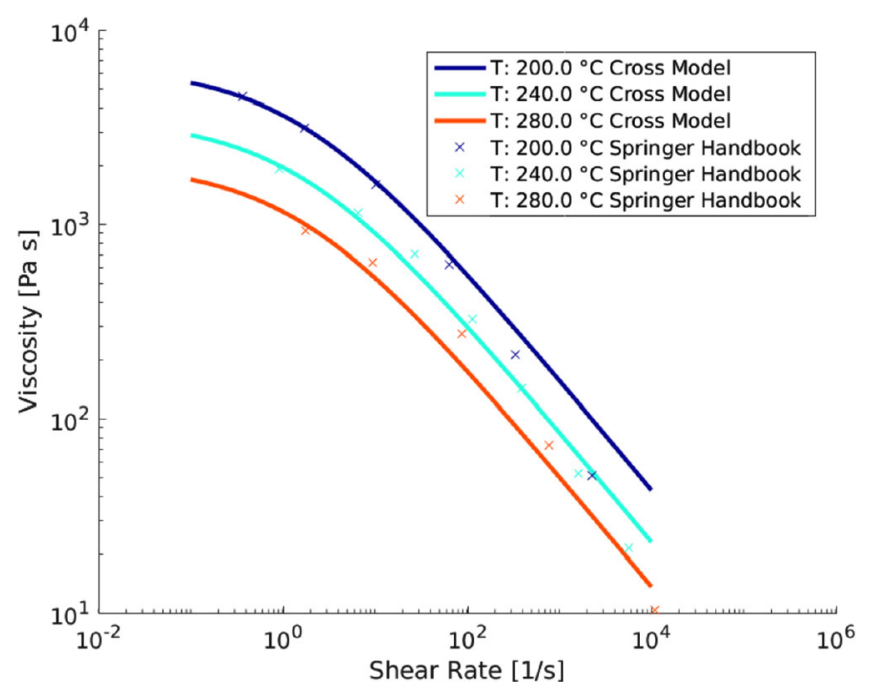

Figure 6 Viscosity of Polypropylene (PP) from Springer Handbook of Condensed Matter and Materials Data [20, Fig. 3.3-13] and fitted cross model with coefficients from Table 2

Table 2 Cross model coefficients to model the viscosity of Polypropylene (PP) in Fig. 6

\begin{tabular}{lll}
\hline zero-shear-rate viscosity & $\eta_{0}$ & $6421.8 \mathrm{~Pa} \mathrm{~s}$ \\
time constant & $\lambda$ & $0.6304 \mathrm{~s}$ \\
power-law index & $n$ & 0.4276 \\
activation energy & $\alpha$ & $3742.5 \mathrm{~K}$ \\
reference temperature & $T_{\text {alpha }}$ & $473.16 \mathrm{~K}$ \\
\hline
\end{tabular}

$\rho=900 \mathrm{~kg} \mathrm{~m}^{-3}$ [20, Table 3.3-5] and a constant temperature of $T=237^{\circ}$ was used. The inertial resistance coefficient for the filter was set to $C_{\text {filter }}=1 \mathrm{e}+13 \mathrm{~m}^{-1}$. For the applied mass flow rate this results in a pressure drop of about 5 bar through the filter. At the inlet a constant mass flow of $41 \mathrm{~kg} \mathrm{~h}^{-1}$ was set. The velocity profile itself is then determined by the solver. At all outlets of the 279 capillaries an outflow boundary condition with zero ambient pressure was set. At the boundaries of the porous Filter a slip condition was used because friction is already represented in the source term. No-slip boundary conditions were used on all other boundaries.

Numerical results. Simulations for the given setup for the Full Model were performed with $\mathrm{ANSYS}^{\circ}$ Fluent for the reference design, as well as for the two optimized designs (Optimized A and Optimized B). Results for the wall shear stress in Cavity 1 are shown in Fig. 7. The wall shear stress in the reference design Fig. 7(a) is very low, especially in the outer regions which indicates stagnation zones. The wall shear stress for the optimized designs in Fig. 7(b) (Optimized A) and Fig. 7(c) (Optimized B) is significantly higher. As intended by the shape optimization approach the wall shear stress is very uniform. This shows that the shapes obtained with the Surrogate Model are still valid with the Full Model. Note, that the absolute level of the wall shear stress differs between the optimization step (Figs. 3 and 4) and the validation step (Fig. 7). The reason is that the optimization step was carried out in a dimensionless setting, while typical real world values were used for the validation step.

In order to quantify and compare the residence time we compute a representative number of pathlines through the spin pack (cf. (5)). Specifically the 279 pathlines which end in 


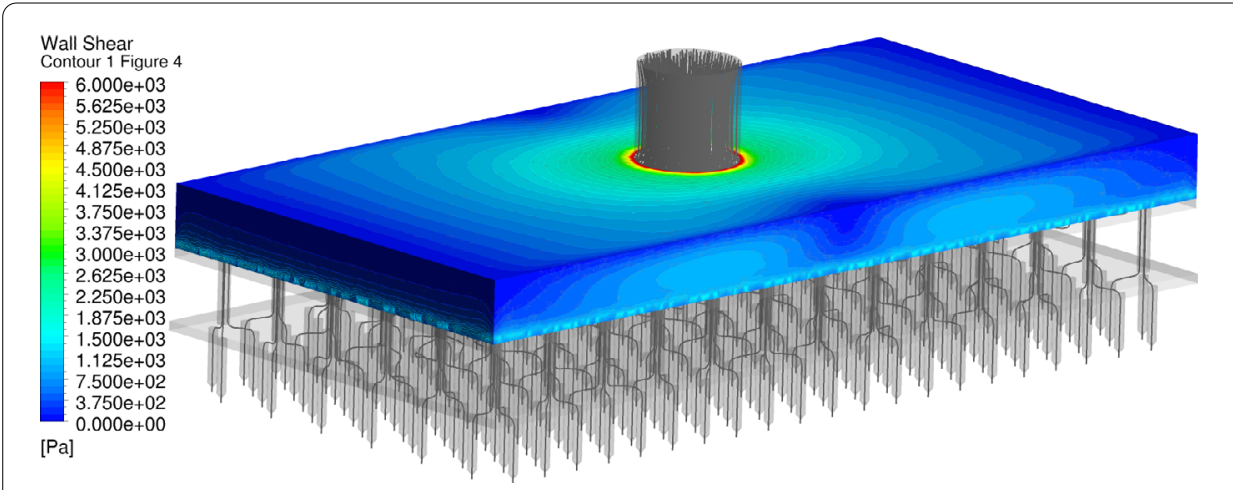

(a) Reference

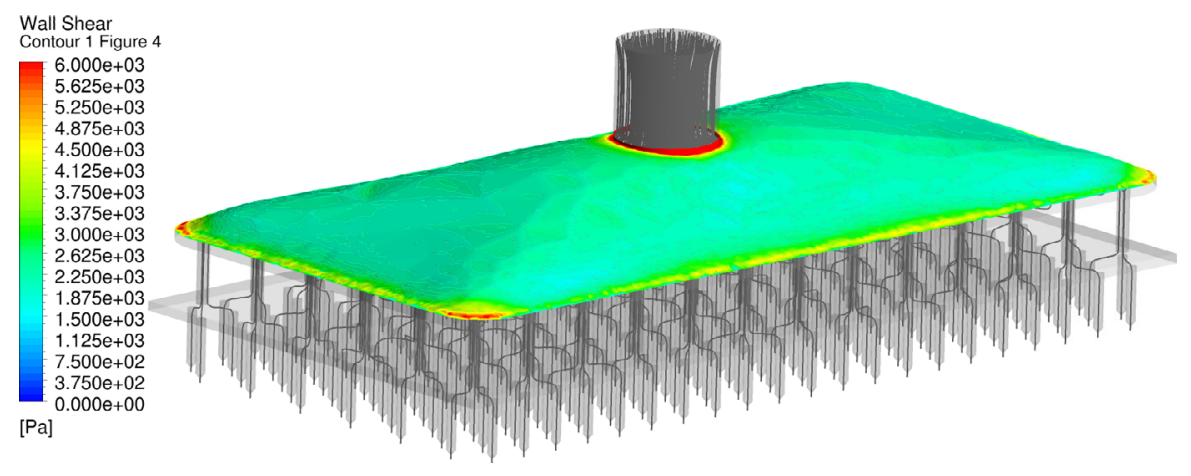

(b) Optimized A

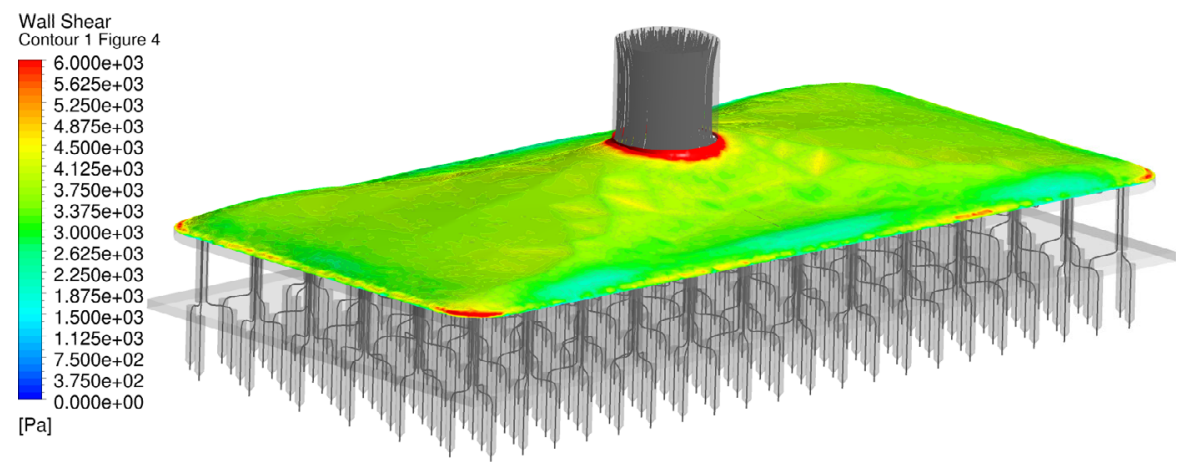

(c) Optimized B

Figure 7 Comparison of the wall shear stress in Cavity 1 for the three spin pack designs. Furthermore, the 279 pathlines which end in the center of each capillary are shown

the centers of each capillary outlet were traced back in time along the velocity field until they reach the inlet. These pathlines are also depicted in Fig. 7. For each pathline the total residence time spent in the whole spin pack is measured as well as the residence time for each component. Figure 8 shows a box plot of the residence times for the three design variations. The black horizontal line in the center of the colored region represents the median. The colored region reaches from the $25 \%$-quantile to the $75 \%$-quantile and the whiskers from the $5 \%$-quantile to the $95 \%$-quantile. The rightmost boxes show the total residence time spent in the spin pack while the others are the breakdown to the individual components. For the reference design most of the residence time is spent in Cavity 1 . The 


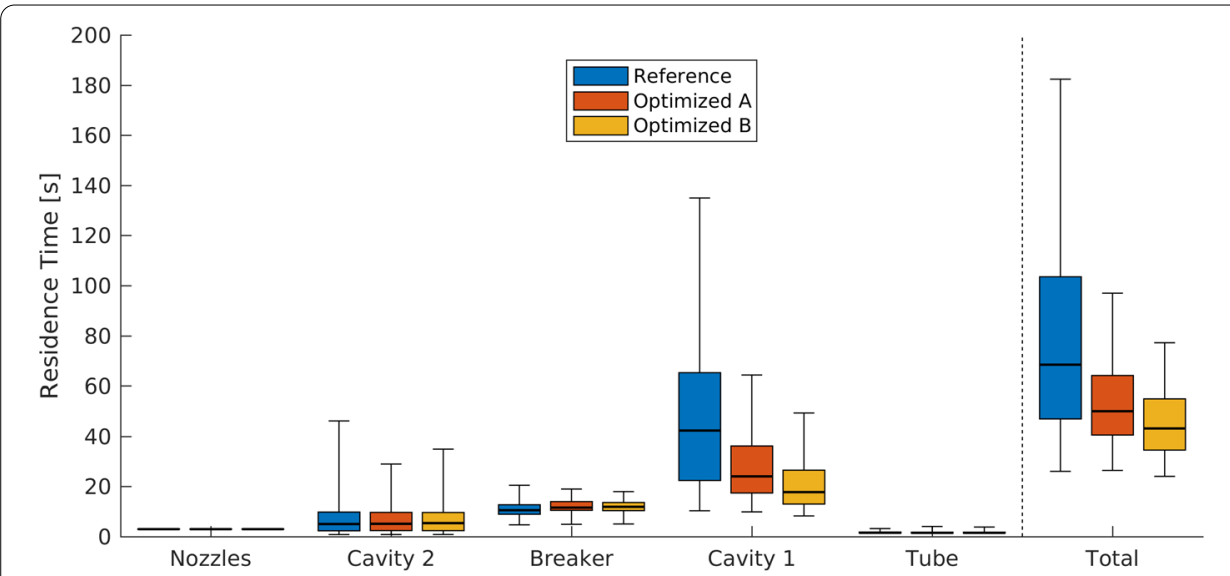

Figure 8 Comparison of the residence times measured along 279 representative pathlines for the three geometric design variations

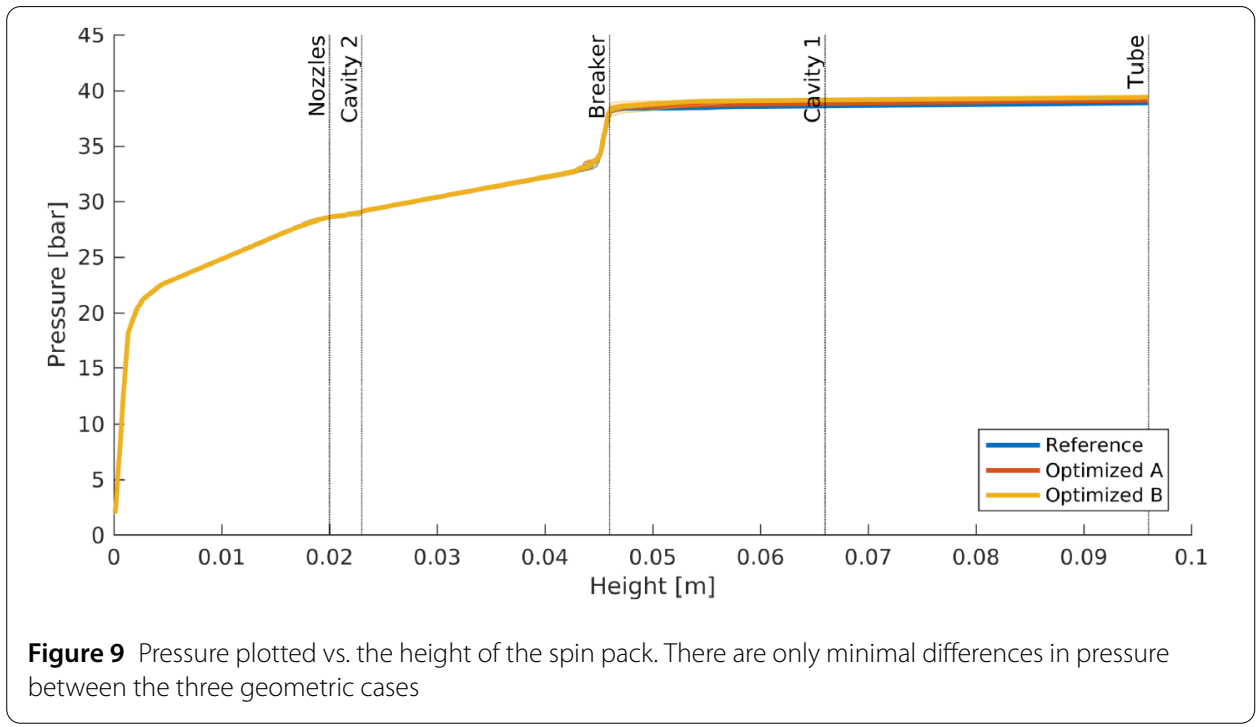

optimized designs show a significant reduction of the residence time in Cavity 1 as well as for the total time. Also the spread of residence time was greatly reduced.

Before we end our investigation we check the level of pressure. Naturally, an increased wall shear stress results in an increased pressure drop through the spin pack. Not to cause any other problems we have to make sure that this additional pressure drop stays moderate. Again we use the pathlines to plot the pressure. Figure 9 shows the pressure plotted vs. the height direction. The pressure is nearly constant in each layer with equal height. So there are no visible differences between the individual pathlines for the same geometric case. But also the pressure differences between the reference case and the two optimized versions are minimal and only slightly visible in the Cavity 1 and Tube region. Furthermore, we see that the main pressure drop is generated by the fine capillaries, followed by the Filter which sits at the top of the Breaker Plate. The two cavities are only responsible for a very small drop in pressure. 


\section{Conclusion}

The presented shape optimization approach is able to generate cavities with specific wall shear stress profiles. This has proven to be an effective tool in the optimization of polymer spin packs and filter devices for sensitive applications. It is now possible to design geometries without dead spaces and with short and more uniform residence times. The optimization approach uses the Surrogate Model as a simplification. However, the validation has shown that the cavities optimized with the simplified model are still valid in the more realistic setting of the Full Model. We have further seen that cavities with optimized wall shear stress lead to reduced and more uniform residence times within the spin pack while the increase in pressure stays minimal. Our ongoing and future research on this matter includes the use of parameterized geometries to improve the regularity as well as optimizing the residence time directly without the use of wall shear stress as an indirect criterion.

\section{Acknowledgements}

Not applicable.

Funding

This work was supported by the German Federal Ministry of Education and Research (BMBF) grant no. 03MS606F and by the German Federal Ministry for Economic Affairs and Energy (BMWI) grant no. IGF 17629 N.

\section{Abbreviation}

Not applicable.

Availability of data and materials

All spin pack geometries depicted in Fig. 7 are provided.

\section{Competing interests}

The authors declare that they have no competing interests.

\section{Authors' contributions}

All authors read and approved the final manuscript.

\section{Author details}

${ }^{1}$ Fraunhofer Institute for Industrial Mathematics ITWM, Kaiserslautern, Germany. ${ }^{2}$ TU Kaiserslautern, Kaiserslautern, Germany.

\section{Publisher's Note}

Springer Nature remains neutral with regard to jurisdictional claims in published maps and institutional affiliations.

Received: 12 February 2018 Accepted: 27 November 2018 Published online: 04 December 2018

\section{References}

1. Leithäuser C, Feßler R. Characterizing the image space of a shape-dependent operator for a potential flow problem. Appl Math Lett. 2012;25(11):1959-63.

2. Leithäuser C. Controllability of Shape-dependent Operators and Constrained Shape Optimization for Polymer. Distributors [PhD Thesis]. Kaiserslautern: TU; 2013.

3. Leithäuser C, Pinnau R, Feßler R. Approximate controllability of linearized shape-dependent operators for flow problems. ESAIM Control Optim Calc Var. 2017;23(3):751-71.

4. Leithäuser C, Pinnau R. The production of filaments and non-woven materials: the design of the polymer distributor. In: Math for the digital factory. Berlin: Springer; 2017. p. 321-40

5. Quarteroni A, Rozza G. Optimal control and shape optimization of aorto-coronaric bypass anastomoses. Math Models Methods Appl Sci. 2003;13(12):1801-24.

6. Rozza G. On optimization, control and shape design of an arterial bypass. Int J Numer Methods Fluids. 2005:47(10-11):1411-9.

7. ANSYS ${ }^{\oplus}$ Fluent, Release 18.1, Help System, Fluent Guide;

8. Kennedy P, Zheng R. Flow analysis of injection molds. Munich: Hanser Verlag; 2013.

9. Nield DA, Bejan A. Convection in porous media. New York: Springer; 2006.

10. Ern A, Guermond JL. Theory and practice of finite elements. vol. 159. Berlin: Springer; 2004.

11. Simon J. Differentiation with respect to the domain in boundary value problems. Numer Funct Anal Optim. 1980;2(7):649-87.

12. Amrouche C, Girault V. On the existence and regularity of the solution of Stokes problem in arbitrary dimension. Proc Jpn Acad, Ser A, Math Sci. 1991;67(5):171-5. 
13. Wloka J. Partial differential equations. Cambridge: Cambridge University Press; 1987.

14. Sokolowski J, Zolesio JP. Introduction to shape optimization: shape sensitivity analysis. vol. 16. Berlin: Springer; 1992.

15. Mohammadi B, Pironneau O. Applied shape optimization for fluids. USA: Oxford University Press; 2001.

16. Alt W. Nichtlineare Optimierung. Wiesbaden: Vieweg; 2002

17. Kroon DJ. Smooth Triangulated Mesh. http://www.mathworks.com/matlabcentral/fileexchange/ 26710-smooth-triangulated-mesh (2010).

18. Desbrun M, Meyer M, Schröder P, Barr AH. Implicit fairing of irregular meshes using diffusion and curvature flow. In: Proceedings of the 26th annual conference on computer graphics and interactive techniques. New York: ACM; 1999. p. 317-24.

19. Hinze M, Pinnau R, Ulbrich M, Ulbrich S. Optimization with PDE constraints. vol. 23. Netherlands: Springer; 2009.

20. Martienssen W, Warlimont H. Springer handbook of condensed matter and materials data. Berlin: Springer; 2006.

\section{Submit your manuscript to a SpringerOpen ${ }^{\circ}$} journal and benefit from:

- Convenient online submission

Rigorous peer review

Open access: articles freely available online

High visibility within the field

- Retaining the copyright to your article

Submit your next manuscript at $\gg$ springeropen.com 